\title{
Spectra of Tensor Operators Adapted to Nonstandard Basis. Qualitative Features of Clustering
}

\author{
BORIS I. ZHILINSKII \\ Laboratoire de Spectronomie Moléculaire, Université de Dijon, 21000 Dijon, France, and \\ Molecular Spectroscopy Laboratory, Department of Chemistry, \\ Moscow State University, Moscow 117234 USSR $^{1}$
}

\begin{abstract}
Spectra of irreducible tensor operators for the chain of group $G \subset O(3)$ are investigated from the qualitative point of view. Conditions are found for clustering of the eigenvalues of tensor operators in the high- $J$ limit. Approximate formulas are proposed which permit the calculation of the relative positions of the clusters. Centrifugal distortion of spherical tops, crystal-field splitting, and low-frequency bending vibrations of nonrigid molecules composed of an atom and rigid symmetrical core are considered as physical examples. Clustering of the $F^{(k J)}$ coefficients of Moret-Bailly are qualitatively explained as well.
\end{abstract}

\section{INTRODUCTION}

Recent analyses of high-resolution infrared spectra of $\mathrm{SF}_{6}$ and $\mathrm{CH}_{4}(1,2)$ have shown the existence of intriguing cluster patterns at rather high $J$ values and have focused interest on the theoretical description of such clustering (3-5). From the mathematical point of view the calculation of rotational level splitting caused by centrifugal distortion of highly symmetrical molecules is rather simple and may be achieved by the diagonalization of a tensor operator (or a linear combination of operators) invariant with respect to the symmetry group $G$ of the molecule considered. For example, the centrifugal splitting of $\mathrm{SF}_{6}$ rotational levels is described in the first approximation by the spectrum of an octahedrally invariant fourth-rank tensor operator $(6,7)$. The results of many numerical calculations establish unambiguously the existence of clustering in the high- $J$ limit $(3,4,8-10)$. The first theoretical interpretation of this phenomenon was given by Dorney and Watson (9) in their $\mathrm{CH}_{4}$ calculations. Classical mechanical arguments were used to explain the qualitative features of tetrahedrally invariant fourth-rank tensor operator. The same clustering may be found in the tables of $F^{(4 J)}$ coefficients by Moret-Bailly et al. (8). It is mainly because of the existence of clustering that approximate algebraic expressions for $F^{(4 J J)}$ coefficients were obtained (II). Recently two independent and extensive investigations were performed by Fox et al. (3) and Harter and Patterson (5). The first group of authors calculated the $F^{(4 J J)}$ coefficients of Moret-Bailly for $J \leqslant 100$ and have described the regularities observed in their computer data. On the other hand, Harter and Patterson proposed both qualitative and quantitative arguments for the clustering in the spectra of the fourth-rank tensor operator.

\footnotetext{
${ }^{1}$ Permanent address.
} 
The aim of the present paper is to give the general qualitative theory of the orbital level splitting caused by any tensor operator invariant with respect to group $G$ which is the subgroup of $S O(3)$ or $O(3)$. Some preliminary results were presented in an earlier, short note (12).

The rotational level splitting is not the only problem to which the theory described below may be applied; this problem is the simplest, however, because only tensor operators which are diagonal in rotational quantum number must be considered $(6,7)$. Crystal-field theory provides an example (13) in which nondiagonal tensor operators must also be taken into account. It will be shown that the nondiagonal tensor operators result in the same clustering of the eigenvalues as diagonal ones, but only in the weak coupling case. The structure of the spectrum changes significantly in the case of intermediate coupling. Thus it is no wonder that the experimental observation of clusters was realized during the study of rotational spectra and not in the study of higher- $J$ atomic-level splitting in the cubic crystal field (see comments in Ref. (5)). A third physical problem, which is closely related to the crystal-field theory, is the description of low-frequency spectra of some inorganic nonrigid molecules composed of a rigid symmetrical core and one or several atoms (ions) which move almost freely around this core. Large numbers of ionic molecules are appropriate examples (14-16). It is in fact the general problem of the theoretical description of gas-phase nonrigid molecule spectra that stimulated the research presented here. But the results obtained are formulated in a rather general form, applicable to different physical problems.

This work is organized as follows. In Section II the qualitative structure of equivalent tensor operators diagonal in $J$ is discussed and the relation to the qualitative theory of $F^{(4 J N}$ coefficients is shown. The close connection with the approach of Harter and Patterson (5) is discussed. Some simple formulas for calculation of the cluster positions are given as well. In Section III, the case of nondiagonal tensor operators is investigated. The third-order tensor operator for the chain $T_{d} \subset O(3)$ is considered in detail because of the importance of this operator for describing the fine structure of $A B X_{4}$ nonrigid molecule spectra. Some physical applications are discussed briefly in Section IV, and the last section is devoted to some possible further developments of the theory.

\section{SPECTRA OF EQUIVALENT DIAGONAL TENSOR OPERATORS}

\section{A. Preliminaries}

The mathematical formulation of the problem involves finding the eigenvalues of the operator

$$
T^{(0)}+\lambda T_{p}^{(k)},
$$

where $T^{(0)}$ is the zero-order irreducible spherical tensor operator: Its usual physical realization is the total angular momentum operator. $T_{p}^{(k)}$ is the $k$-rank tensor operator transforming according to the irreducible representation of the group $G$ which is the symmetry group of the problem considered. Index $p$ characterizes the nonstandard basis; $p=(n, C, \sigma)$, where $C$ is the irreducible representation of $G, \sigma$ is the row, and $n$ is the multiplicity label. Although this notation of Moret-Bailly (7) has until now been used by spectroscopists to indicate eigenvalues or eigenfunc- 
tions of $T_{A_{1}}^{(4)}$ (the so-called superadapted basis), it will be used here for any symmetry-adapted basis, in particular for the basis which diagonalizes a given highorder tensor operator.

If $G$ is not a subgroup of $S O(3)$ but only a subgroup of $O(3)$, it is necessary to generalize the notation slightly by introduction of the parity index $\alpha$, which may be $u$ or $g$. Such an extension is necessary, for example, for group $T_{d}$, where operator (1) must be written as

$$
T^{(0, g)}+T_{D}^{(k, \alpha)} .
$$

Usually only $G$-invariant tensor operators are physically meaningful. So index $p$ corresponds to the totally symmetric representation of group $G$.

Two different cases are to be considered: (i) Operator $T_{p}^{(k)}$ is diagonal in $J$ ( $J$ is the quantum number characterizing different energy levels of the zero-order operator). (ii) Operator $T_{p}^{(k)}$ is not diagonal in $J$.

The first case will be considered in this section. The problem can be treated separately for each $J$ value. Moreover, for $J$ fixed, operator $T^{(0)}$ is a multiple of the identity operator and so the problem is to diagonalize operator $T_{p}^{(k)}$ for some $J$ value. The value of $\lambda$ is not important in such a case. This mathematical model is applicable for strictly diagonal operators and for arbitrary operators in the case of the weak coupling limit, i.e., where first-order perturbation theory is adequate to treat the problem (e.g., where the splitting of each degenerate energy level of operator $T^{(0)}$ is small compared with the differences between zero-order states). It is to be noted that there also exist cases for which the first-order perturbation treatment yields no splitting. The tetrahedrally invariant third-rank tensor operator is an appropriate example. All diagonals in $J$ matrix elements of this operator are identically equal to zero. Thus even in the weak coupling limit the method of the diagonal equivalent tensor operator is not applicable. This more complicated problem will be treated in the following section.

We develop here the algebraic treatment of tensor operators which is formally equivalent to the selection of basis functions made by Harter and Patterson (5). The approach based on the commutativity relations between tensor operators is more general than the original version of Harter and Patterson because of the applicability to nondiagonal as well as to diagonal tensor operators. Moreover, it gives the prescription as to how to find the basic functions describing the clustered states without any reference to the form of the potential surface related to the tensor operator considered. We will show that the form of the tensor operator is sufficient to predict the existence of clustering.

To explain the qualitative aspects of the energy spectra for diagonal tensor operators, consider now the relations between operator $T_{p}^{(k)}$ and operator $J_{z}$. The different orientations of the $z$ axis with respect to molecular symmetry axes are to be considered. The transformation of the operators to different axes is essential for both methods, the present one and that of Harter and Patterson. But the reason for such a transformation is quite different. Harter and Patterson do such a transformation after the choice of the basis functions, whereas here the comparison of the different operator forms is necessary to predict the existence of clustering.

Let the tensor operator $T_{p}^{(k)}$ be expressed in terms of the standard basis components with some particular orientation of the $z$ axis as follows: 


$$
T_{p}^{(k)}=\sum_{M_{A}=-k}^{k}{ }^{(k)} G_{p}^{M_{\beta}} T_{M_{\beta}}^{(k)},
$$

where ${ }^{(k)} G_{p}^{M_{B}}$ is the transformation matrix from the standard basis with the $z$ axis oriented in the $\beta$ direction to the basis adapted to subgroup $G \subset S O(3)$. Extension to the case $G \subset O(3)$ is trivial. The explicit form of the tensor operator depends on the choice of the quantization axis. Below, the invariant tensor operators for group $T_{d}$ of third, fourth, sixth, and eighth orders will be considered as examples; different expressions for these operators are listed here for reference. If the $z$ axis coincides with the fourfold symmetry axis of group $T_{d}$, then

$$
\begin{aligned}
& T_{A_{1}}^{3 w)}\left(z=S_{4}\right)=(-i / 2)\left(V_{2}^{(3)}-V_{-2}^{(3)}\right), \\
& T_{A_{1}}^{(q)}\left(z=S_{4}\right)=(7 / 12)^{1 / 2} V_{0}^{(4)}+(5 / 24)^{1 / 2}\left(V_{4}^{(4)}+V_{-4}^{(4)}\right), \\
& T_{A_{1}}^{(6 g)}\left(z=S_{4}\right)=-(1 / 8)^{1 / 2}\left[V_{0}^{(6)}-(7 / 2)^{1 / 2}\left(V_{4}^{(6)}+V_{-4}^{(6)}\right)\right], \\
& T_{A_{1}}^{(8 g)}\left(z=S_{4}\right)=\frac{(33)^{1 / 2}}{8} V_{0}^{(8)}+\frac{(42)^{1 / 2}}{24}\left[V_{4}^{(8)}+V_{-4}^{(8)}\right]+\frac{(390)^{1 / 2}}{48}\left[V_{8}^{(8)}+V_{-8}^{(8)}\right],
\end{aligned}
$$

whereas for the case of the threefold axis as the quantization one, we have

$$
\begin{aligned}
& T_{A 1}^{(3 u)}\left(z=C_{3}\right)=(5 / 9)^{1 / 2} V_{0}^{(3)}+(2 / 9)^{1 / 2}\left(V_{3}^{(3)}-V_{-3}^{(3)}\right), \\
& T_{A_{1}}^{(49)}\left(z=C_{3}\right)=-(2 / 3)\left[(7 / 12)^{1 / 2} V_{0}^{(4)}+2(5 / 24)^{1 / 2}\left(V_{-3}^{(4)}-V_{3}^{(4)}\right)\right] \text {, } \\
& T_{A_{1}}^{(6)}\left(z=C_{3}\right)=-(1 / 8)^{1 / 2}\left[(16 / 9) V_{0}^{(6)}+(2 / 9)(70 / 3)^{1 / 2}\left(V_{3}^{(6)}-V_{-3}^{(6)}\right)\right. \\
& \left.+(2 / 9)(77 / 3)^{1 / 2}\left(V_{6}^{(6)}+V_{-6}^{(6)}\right)\right] . \\
& T_{A_{1}}^{(8 g)}\left(z=C_{3}\right)=\frac{(33)^{1 / 2}}{27} V_{0}^{(8)}-\frac{2(35)^{1 / 2}}{27}\left[V_{3}^{(8)} \cdots V_{-3}^{(8)}\right]+\frac{4(13)^{1 / 2}}{27}\left[V_{6}^{(8)}+V_{-6}^{(8)}\right]
\end{aligned}
$$

The expressions become more complicated if one uses the $C_{2}$ symmetry axis for group $O$.

$$
\begin{aligned}
& T_{A 1}^{(4)}\left(z=C_{2}\right)=-(1 / 4)\left[(7 / 12)^{1 / 2} V_{0}^{(4)}-(35 / 6)^{1 / 2}\left(V_{2}^{(4)}+V_{-2}^{(4)}\right)\right. \\
& \left.-(15 / 8)^{1 / 2}\left(V_{4}^{(4)}+V_{-4}^{(4)}\right)\right], \\
& T_{A 1}^{(6)}\left(z=C_{2}\right)=\frac{13(2)^{1 / 2}}{32} V_{0}^{(6)}+\frac{(210)^{1 / 2}}{64}\left[V_{2}^{(6)}+V_{-2}^{(6)}\right] \\
& -\frac{5(7)^{1 / 2}}{32}\left[V_{4}^{(6)}+V_{-4}^{(6)}\right]+\frac{(462)^{1 / 2}}{64}\left[V_{6}^{(6)}+V_{-6}^{(6)}\right], \\
& T_{A_{1}}^{(8 g)}\left(z=C_{2}\right)=\frac{9(33)^{1 / 2}}{128} V_{0}^{(8)}-\frac{(1155)^{1 / 2}}{192}\left[V_{2}^{(8)}+V_{-2}^{(8)}\right] \\
& +\frac{25(42)^{1 / 2}}{384}\left[V_{4}^{(8)}+V_{-4}^{(8)}\right]+\frac{7(13)^{1 / 2}}{64}\left[V_{6}^{(8)}+V_{-6}^{(8)}\right]+\frac{3(390)^{1 / 2}}{256}\left[V_{8}^{(8)}+V_{-8}^{(8)}\right] .
\end{aligned}
$$

Some of these equations are surely well known (see, for example, Ref. (5)). 
To study the relations between the eigenfunctions of $T_{p}^{(k)}$ and the standard basis functions, consider the commutator

$$
\left[J_{0}, T_{p}^{(k)}\right]=\sum_{M_{\beta}=-k}^{k} M_{\beta}{ }^{\left({ }^{k}\right)} G_{p}^{M_{\beta}} T_{M_{\beta}}^{(k)} .
$$

The symbol $J_{0}$ is used instead of $J_{z}$ because of ambiguity in the $z$-axis choice. If there exist some functions which are the eigenfunctions of both $T_{p}^{(k)}$ and $J_{0}$ then the corresponding rows and columns of the matrix representing the commutator in the standard basis must be identically zero, because standard basis functions are eigenfunctions of operator $J_{0}$. Below, the term "good commutativity of operators $A$ and $B$ in subspace $M$ " will be used, which means (in analogy with "good quantum numbers") that operators $A$ and $B$ may be simultaneously transformed to a form such that all nondiagonal (with respect to $\mathfrak{M}$ ) matrix elements are negligible compared with diagonal ones. It will be shown that it is precisely the "good commutativity" of $T_{p}^{k)}$ and $J_{0}$ in certain subspaces which is responsible for the formation of clusters in the spectra of irreducible tensor operators; i.e., these basis functions are just the ones which must be used in Harter-Patterson's treatment to induce the clustered functions. So the index $M$ is the good quantum number for the cluster labeling. To demonstrate good commutativity it is necessary to calculate the matrix elements of the commutator in the standard basis. To construct the diagonal equivalent tensor operator we use the well-known procedure

$$
\begin{aligned}
T_{k}^{(k)} & =(-1)^{k}[(2 k) ! / k ! k !]^{1 / 2}\left(J_{+}\right)^{k}, \\
T_{\mu}^{(k)} & =[(k+\mu) !(2 k) !(k-\mu) !]^{1 / 2}\left[J_{-}, T_{k}^{(k)}\right]_{(k-\mu)}, \\
{[A, B]_{(0)} } & =B, \quad[A, B]_{(1)}=[A, B] ; \quad[A, B]_{(2)}=[A,[A, B]], \ldots .
\end{aligned}
$$

Only the asymptotic behavior of the matrix elements for the high- $J$ limit is necessary for further use and not the numerical factors. So it is sufficient to know the powers of operators $J_{+}, J_{0}$, and $J^{2}$ in the general expression of the equivalent operator $T_{\mu}^{(k)}$. The general structure follows immediately from the commutation relations

$$
\begin{aligned}
{\left[J_{-}, J_{0}^{k} J_{+}^{t}\right]=\{J(J+1)} & +\sum_{n=1}^{k-1}\left[\left(\begin{array}{l}
k \\
n
\end{array}\right) J(J+1)-\left(\begin{array}{l}
k+1 \\
n-1
\end{array}\right)\right] J_{0}^{n} \\
& \left.+\left[t(t-1)-\frac{(k+1) k}{2}\right] J_{0}^{k}+(-2 t-k) J_{0}^{k+1}\right\}_{+}^{t-1} .
\end{aligned}
$$

It can easily be seen that for $\mu>0$, operator $T_{\mu}^{(k)}$ includes $J_{+}^{\mu}$ multiplied maximally by $J_{0}^{k-\mu}$ or $[J(J+1)]^{(k-\mu) / 2}$ for even $(k-\mu)$ and $[J(J+1)]^{(k-\mu-1) / 2} J_{0}$ for odd $(k-\mu)$. It is convenient to use instead of $T_{\mu}^{(k)}$ some new operator obtained from $T_{\mu}^{(k)}$ upon multiplying by $\left[(2 J-k+1)_{2 k+1}\right]^{1 / 2}$, where $(\alpha)_{\beta}=\alpha(\alpha+1)(\alpha+2) \ldots$ $(\alpha+\beta)$. This factor is simply the inverse reduced matrix element of operator $T_{\mu}^{i k)}$ in the $J$ basis. The calculation is now straightforward if one uses the formula

$$
\left\langle J M+\mu\left|J_{+}^{\mu}\right| J M\right\rangle=\left[\frac{[J-M) !(J+M+\mu) !}{(J-M-\mu) !(J+M) !}\right]^{1 / 2},
$$




$$
\begin{aligned}
\lim _{J \rightarrow \infty} \frac{\left\langle J M+\mu\left|T_{\mu}^{(k)}\right| J M\right\rangle}{\left[(2 J-k+1)_{2 k+1}\right]^{1 / 2}} & \\
& \sim(J)^{-(2 k+1) / 2}\left[\frac{(J+M+\mu) !(J-M) !}{(J-M-\mu) !(J+M) !}\right]^{1 / 2}\left[c_{1}(M+\mu)^{k-\mu}\right. \\
& \left.+\left\{\begin{array}{ll}
c_{2}[J(J+1)]^{(k-\mu) / 2} & \text { for }(k-\mu) \text { even } \\
c_{3}(M+\mu)[J(J+1)]^{(k-\mu-1) / 2} & \text { for }(k-\mu) \text { odd }
\end{array}\right\}+\cdots\right]
\end{aligned}
$$

Here the $c_{i}$ are numerical constants. Two particular cases of Eq. (19) are of great importance: those corresponding to $M+\mu=J$ and those corresponding to $M+\mu=0$.

For $M+\mu=J$ and $J \gg \mu$,

$$
\lim _{J \rightarrow \infty}\left[\frac{(J-M) !(J+M+\mu) !}{(J-M-\mu) !(J+M) !}\right]^{1 / 2}=\frac{(\mu !)^{1 / 2}}{2^{\mu / 2}}(J)^{\mu / 2},
$$

and for $M+\mu=0$

$$
\lim _{J \rightarrow \infty}\left[\frac{(J-M) !(J+M-\mu) !}{(J-M-\mu) !(J+M) !}\right]^{1 / 2}=(J)^{\mu} .
$$

Thus for $M+\mu=J$ the general formula (19) may be simplified to

$$
\lim _{J \rightarrow \infty} \frac{\left\langle J J\left|T_{\mu}^{(k)}\right| J J-\mu\right\rangle}{\left[(2 J-k+1)_{2 k+1}\right]^{1 / 2}} \sim(J)^{-(\mu+1) / 2},
$$

and in a similar way for $M+\mu=0$,

$$
\lim _{J \rightarrow \infty} \frac{\left\langle J 0\left|T_{\mu}^{(k)}\right| J-\mu\right\rangle}{\left[(2 J-k+1)_{2 k+1}\right]^{1 / 2}} \sim \begin{cases}(J)^{(\mu-k-1) / 2} & \text { for }(k-\mu) \text { even } \\ (J)^{(\mu-k-2) / 2} & \text { for }(k-\mu) \text { odd. }\end{cases}
$$

To facilitate understanding, the asymptotics of the matrix elements for some particular cases of tensor operators, $T_{\mu}^{(k)}, k=4, \mu=0-4$, are shown in Fig. 1 . The commutators of $T_{\mu}^{(k)}$ with $J_{0}$ have the same asymptotic behavior as the $T_{\mu}^{k)}$ (except for $T_{0}^{(k)}$, which commutes strictly with $J_{0}$ ). From (22) and (23) it follows that good commutativity exists between operator $T_{k}^{(k)}$ and $J_{0}$ in the subspace $M=J$. The corresponding nondiagonal matrix elements are significantly smaller in this subspace than in any others. In contrast operator $T_{1}^{(k)}$ possesses good commutativity with $J_{0}$ in the subspace $M=0$. This means, for example, that operator $T_{4}^{(4)}$ has eigenfunctions which may be approximated well by the $|J, M=J\rangle$ standard basis functions. That is, the second-order term in Eq. (11) on p. 4887 of Ref. (5) may be negligible. On the other hand the $|J, M=0\rangle$ standard basis function seems to be a good approximation for some eigenfunction of operator $T_{1}^{(6)}$, for example.

The same intuitive arguments are applicable to the study of the relations between the eigenfunctions of any $T_{p}^{(k)}$ operator and standard basis functions; but to reach more rigorous results first-order perturbation theory may be applied to tensor operators diagonal in $J$. In such a case the diagonal part of operator $T_{p}^{(k)}$ (i.e., $V_{0}^{(k)}$ standard component) is the zero-order operator, and the part of $T_{p}^{(k)}$ non- 
diagonal in the standard basis is treated as the perturbation. Nondegenerate perturbation theory may be used if the differences between the diagonal matrix elements are large compared with the nondiagonal ones. From Eqs. (22) and (23) it is seen that it is not the case for $M \approx 0$, because not only the differences but the absolute values of the diagonal matrix elements are smaller than the nondiagonal ones. Figure 1 shows this clearly for fourth-rank tensor operators. Simple calculations show that for $M \approx J$ the differences between diagonal matrix elements

$$
\begin{aligned}
& \frac{\left\langle J J\left|V_{0}^{(k)}\right| J J\right\rangle-\left\langle J J-1\left|V_{0}^{(k)}\right| J J-1\right\rangle}{(J \mid k \| J)} \\
& =\left(\begin{array}{ccc}
J & k & J \\
-J & 0 & J
\end{array}\right)-\left(\begin{array}{ccc}
J & k & J \\
-J+1 & 0 & J-1
\end{array}\right) \underset{J \rightarrow \infty}{\rightarrow} \frac{k(k+1)}{(2 J)^{3 / 2}}
\end{aligned}
$$

in the high- $J$ limit must be smaller than nondiagonal elements

$$
\frac{\left\langle J J\left|V_{\mu}^{(k)}\right| J J-\mu\right\rangle}{(J \| k|| J)} \underset{J \rightarrow \infty}{\rightarrow}(J)^{-(\mu+1) / 2}
$$

if only these elements satisfy $\Delta M>2$.

Consider tetrahedrally invariant fourth-order operators as an example. If the quantization axis is the fourfold one, then the diagonal matrix element difference for $M=J$

$$
\left(\begin{array}{ccc}
J & 4 & J \\
-J & 0 & J
\end{array}\right)-\left(\begin{array}{ccc}
J & 4 & J \\
-J+1 & 0 & J-1
\end{array}\right) \underset{J \rightarrow \infty}{\rightarrow} \frac{20}{(2 J)^{3 / 2}}
$$

is larger in the high- $J$ limit than the nondiagonal one

$$
\left(\begin{array}{ccc}
J & 4 & J \\
-J & 4 & J-4
\end{array}\right) \underset{J \rightarrow \infty}{\rightarrow} 35(3)^{1 / 2}(J)^{-5 / 2}
$$

The same situation takes place for tensor operators oriented along the threefold axis, but in this case the difference in the asymptotic behavior is not so pronounced, because the nondiagonal matrix elements are of order $(J)^{-2}$. Finally, for the case of $z$-axis coincidence with the twofold symmetry axis, the order of the nondiagonal matrix elements and the order of the differences between the diagonal elements are the same even for $M=J$. So in such a case the $|J, J\rangle$ basis function has no remarkable properties. It must be noted that perturbation theory may be applicable even in the case of the same asymptotic behavior of the nondiagonal matrix elements and the differences between diagonal ones. Only more accurate investigations which take into account numerical factors, not just the asymptotics, may explain the existence or absence of corresponding clusters for tensor operators including $T_{ \pm 2}^{(k)}$ standard components.

Until now only one fixed standard basis set was considered, and it was shown that some functions from this set are nearly the eigenfunctions of the $T_{p}^{(k)}$ tensor operator. But there exist several equivalent standard basis sets for highly symmetrical molecules. For the $T_{d}$ or $O$ group, for example, there are three equivalent $S_{4}$ axes. Thus, if there exists one standard basis function $|J, J\rangle$ quantized along 

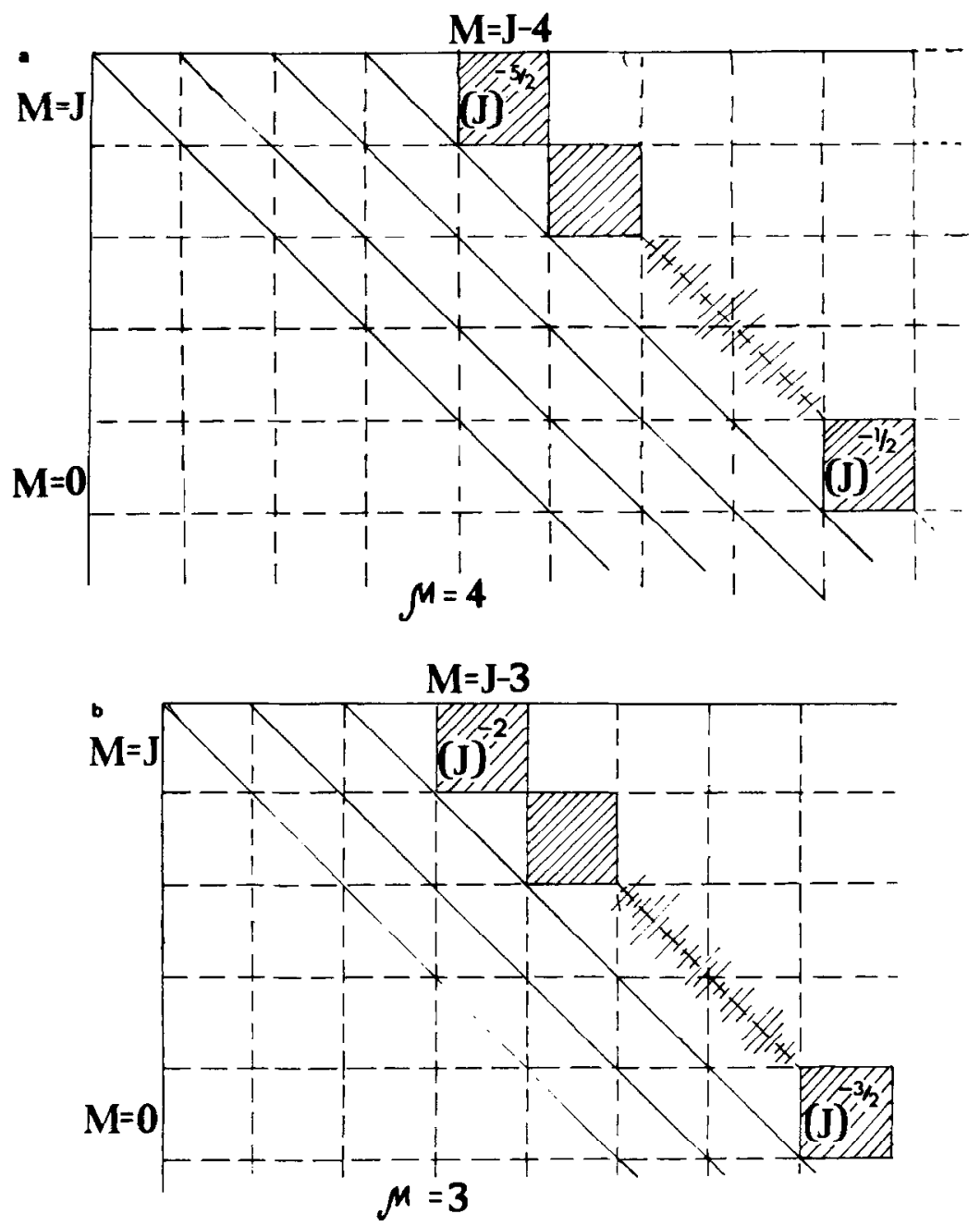

FIG. 1. Schematic representation of $T^{(4)}$ tensor operator components in standard basis. Leading asymptotic terms in the high- $J$ limit are indicated inside small squares. Each matrix has only one diagonal with nonzero elements.

the $S_{4}$ axis, then six functions exist which are approximate eigenfunctions of $T_{p}^{(k)}$ with the same accuracy. These functions correspond to three different but equivalent $S_{4}$ axes and to two orientations for each axis. As noted (5) for the case of $k=4$, first-order energy corrections are strictly the same for all these functions and the first-order perturbation theory results in the clustering of energy levels. It is necessary to verify whether clustering is preserved in second order. Each cluster may be described by the set $|J, M(\gamma)\rangle$ of the standard basis functions with fixed $M$ values but with different equivalent orientations, $\gamma$, of the quantization axis. The order of cluster splitting is given by nondiagonal matrix elements of the $T_{p}^{k}$ ) operator between the standard basis functions $|J, M(\gamma)\rangle$ with the same $M$, but different $\gamma$ : 

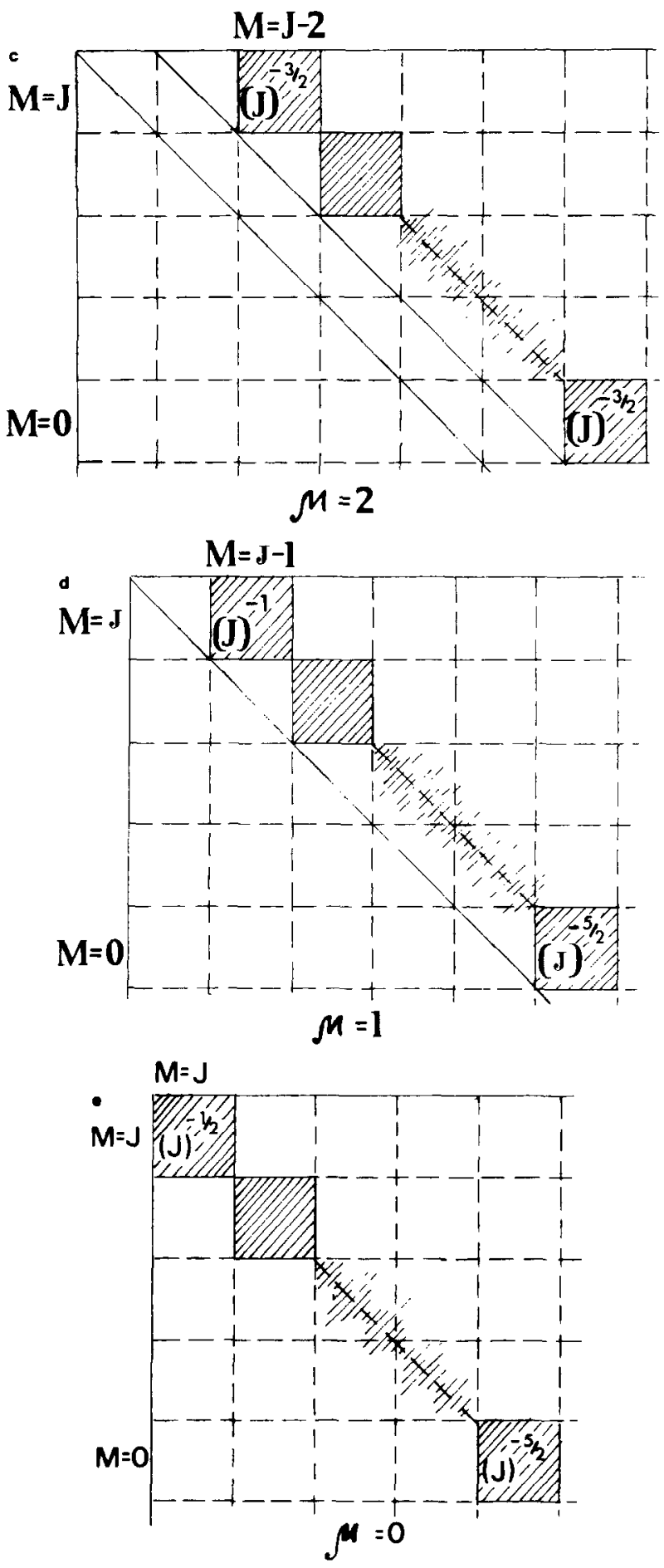

FIG. 1-Continued. 


$$
\left\langle J M(\gamma)\left|T_{p}^{(k)}\right| J M\left(\gamma^{\prime}\right)\right\rangle,
$$

where $\gamma^{\prime}$ is the orientation of a different equivalent axis. The matrix element Eq. (28) for $M=J$ in the high- $J$ limit may be approximated by matrix elements that are nondiagonal in $M$ but diagonal in $\gamma$. To demonstrate this one need only transform $\left|J M\left(\gamma^{\prime}\right)\right\rangle$ into $\left|J M^{\prime}(\gamma)\right\rangle$,

$$
\left|J M\left(\gamma^{\prime}\right)\right\rangle=\sum_{M^{\prime}} D_{M^{\prime} M}^{(J)}\left(\gamma \gamma^{\prime}\right)\left|J M^{\prime}(\gamma)\right\rangle,
$$

where $D^{(\lambda)}\left(\gamma \gamma^{\prime}\right)$ is the matrix of finite rotation which transforms the $\gamma^{\prime}$ axis into the $\gamma$ one; and use the fact that the diagonal matrix element $D_{m M}^{(D)}$ in the high-J limit is negligible compared with nondiagonal ones (19).

So the general idea of this subsection was to show rather intuitively the reasons for cluster formation in the spectra of tensor operators diagonal in $J$. It is clear that the rank of the operator is unimportant and that only types of standard components are essential. Now it is possible to formulate more rigorously the qualitative theory of level splitting and to obtain some approximate formulas for predicting cluster structure of the spectra.

\section{B. Calculation of Cluster Positions}

The general statement concerning equivalent tensor operators is formulated below.

Let $T_{p}^{(k, \alpha)}$ be a tensor operator of rank $k$, which transforms according to the irreducible representation $p=(n, \mathrm{C}, \sigma)$ of group $G \subset O(3)$ and which includes only $V_{\mu}^{(k)}(\mu=0, \pm 3, \pm 4, \ldots)$ standard components for a special choice of quantization axes. Then we have in the high- $J$ limit:

(i) The standard basis functions with $M=J$ are good approximation to the eigenfunctions of $T_{p}^{k, \alpha)}$.

(ii) There exists a group of eigenfunctions with almost degenerate eigenvalues, and the degree of degeneracy is equal to the number of equivalent $z$-axis orientations resulting in the same form of operator $T_{p}^{(k, \alpha)}$

(iii) If there exist several nonequivalent $z$-axis orientations yielding the form of operator $T_{p}^{(k, \alpha)}$ which does not include $V_{ \pm 1}^{(k)}$ and $V_{ \pm 2}^{(k)}$ standard components then the same number of nonequivalent classes of clusters appears in the spectrum of operator $T_{p}^{(k, \alpha)}$.

As an example, octahedrally invariant diagonal tensor operators in the high- $J$ limit necessarily form two types of clusters in their spectra. Sixfold degenerate clusters correspond to six equivalent orientations of 4-fold symmetry axes, while 8-fold degenerate clusters arise from the eight equivalent orientations of 3-fold symmetry axes. Twelvefold degenerate clusters do not always appear in the spectra because, if the quantization axis is directed along 2 -fold symmetry axes, the explicit form of invariant tensor operators includes the $V_{ \pm 2}^{(k)}$ standard components. Crude estimation of the existence of clusters generated by operators which include $V_{ \pm 2}^{(k)}$ but not $V_{ \pm 1}^{(k)}$ standard components is based on the numerical value of the parameter ${ }^{(k)} G_{p}^{2}(\beta) /{ }^{(k)} G_{p}^{0}(\beta)=\lambda$. This parameter characterizes the strength of the perturbation. For $\lambda \geqslant 1$ the clusters must not exist, whereas for 
$\lambda \ll 1$ clustering is possible. For octahedrally invariant tensor operators of orders 4,6 , and 8 the parameter $\lambda$ equals $(10)^{1 / 2},(105)^{1 / 2} / 26$, and $2(35)^{1 / 2} / 27$ correspondingly. So one may expect the existence of 12 -fold clusters for 6 th- and 8 th-order operators and the absence of 12 -fold clusters for the 4th-order tensor operator. Such a suggestion has been verified by numerical investigations performed for a large number of tensor operators invariant with respect to groups $O, T, D_{8}, D_{4}$, and $D_{2}(20)$. It must be noted also that sometimes the tensor operator may take the form satisfying the conditions of clustering even if the $z$ axis does not coincide with any symmetry element. Such a situation was shown to exist for $D_{2}$-invariant tensor operators (20). It probably takes place also for the 10th-order tensor operator. A detailed investigation is now in progress.

Now we describe the procedure which permits calculation of the relative positions of the clusters in the spectra of tensor operators. One may characterize the clusters by label $(\beta, M)$, where $\beta$ is the type of quantization axis which results in the form of tensor operator satisfying conditions for clustering, and $M$ is the angular momentum projection on this axis. Different $\beta$ correspond to different types of clusters, and within the same type the clusters may be distinguished by the $M$ value. For example, for group $T_{d}$ two different $\beta$ are possible, corresponding to a fourfold axis (sixfold clusters) and a threefold axis (eightfold clusters). The possible values of $M$ leading to real clusters are difficult to predict for concrete cases, but some initial values, $M=J, J-1, J-2, \ldots$, are evident. So the problem is to calculate the relative positions of clusters for any diagonal tensor operator $T_{p}^{(k)}$.

The energy of the cluster in the first-order approximation is simply the expectation value of the $T_{p}^{(k)}$ operator calculated with respect to the $|\beta, M\rangle$ trial function; to generalize the $k=4$ results of Ref. (5), the relative positions of clusters may be reduced to a ratio of Clebsch-Gordon coefficients, by means of the WignerEckart theorem

$$
\frac{E(k, J ; \beta, M)}{E\left(k, J ; \beta, M^{\prime}\right)}=\frac{\left\langle\beta, M\left|T_{p}^{(k)}\right| \beta, M\right\rangle}{\left\langle\beta, M^{\prime}\left|T_{p}^{(k)}\right| \beta, M^{\prime}\right\rangle}=\frac{C_{J M k 0}^{J M}}{C_{J M^{\prime} k \theta}^{J M^{\prime}}} .
$$

Particular realizations of (30) for $M=J, J-1, J-2, J-3$ are rather simple. For example,

$$
E(k, J ; \beta, M=J-1)=E(k, J ; \beta, M=J) \frac{2 J-k(k+1)}{2 J},
$$

$E(k, J ; \beta, M=J-2)=E(k, J ; \beta, M=J)$

$$
\times \frac{2(2 J-k)(2 J-k-1)-4 k^{2}(2 J-k)+k^{2}(k-1)^{2}}{4 J(2 J-1)},
$$

$$
\begin{gathered}
E(k, J ; \beta, M=J-3)=\frac{E(k, J ; \beta, M=J)}{12 J(2 J-1)(2 J-2)}[6(2 J-k)(2 J-k-1)(2 J-k-2) \\
\left.-18 k^{2}(2 J-k)(2 J-k-1)+9 k^{2}(k-1)^{2}(2 J-k)-k^{2}(k-1)^{2}(k-2)^{2}\right] .
\end{gathered}
$$

From Eqs. (31)-(33) it can be seen that for $J$ significantly larger than $k^{2}$ the clusters with $M \simeq J$ are situated rather close to the $(\beta, M=J)$ cluster. It means 
that $|E(k, J ; \beta, J)-E(k, J ; \beta, M \approx J)|$ is small compared to the width of the entire manifold. It may be shown that for fixed $k$ and $\delta$ the high- $J$ limit for the energy of cluster $(\beta, M=J-\delta)$ is equal to $L(k, J ; \beta, M=J)$,

$$
\lim _{J \rightarrow \infty} \frac{E(k, J ; \beta, M=J-\delta)}{E(k, J ; \beta, M=J)}=1 .
$$

We can calculate the relative positions of different types of clusters, i.e., the energies of clusters with different $\beta$, as follows. Taking into account formula (3) and utilizing the Wigner-Eckart theorem one has

$$
\frac{E\left(k, J ; \beta_{1}, M\right)}{E\left(k, J ; \beta_{2}, M\right)}=\frac{\left\langle J M_{\beta_{1}}\left|T_{p}^{(k)}\right| J M_{\beta_{1}}\right\rangle}{\left\langle J M_{\beta_{2}}\left|T_{p}^{(k)}\right| J M_{\beta_{2}}\right\rangle}=\frac{{ }^{(k)} G_{p}^{\beta_{1}}}{{ }^{(k)} G_{p}^{\beta_{\beta_{2}}}} .
$$

So the relative positions of the clusters depend only on transformation coefficients from a standard to a nonstandard basis.

As an example, we now construct the qualitative scheme of energy clusters for octahedrally invariant tensor operators. The spectrum of the fourth-rank tensor operator is well known $(4,5,8)$, so the comparison with exact numerical solutions is possible. Figure 2 shows the cluster positions predicted by formulas (30) and (35). Clusters with $\beta=S_{4}$ are sixfold degenerate and $\beta=C_{3}$ clusters are eightfold degenerate. In Table I, the numerical data from Krohn's table (4) are compared with results of simple perturbation calculations. It is seen that the predicted values are significantly more accurate for $S_{4}$ than for $C_{3}$ clusters. Such behavior may be easily explained from the qualitative point of view, upon taking into account Eqs. (5), (9), and (25), which show that nondiagonal matrix elements of $T_{A_{1}}^{(4)}$ in the

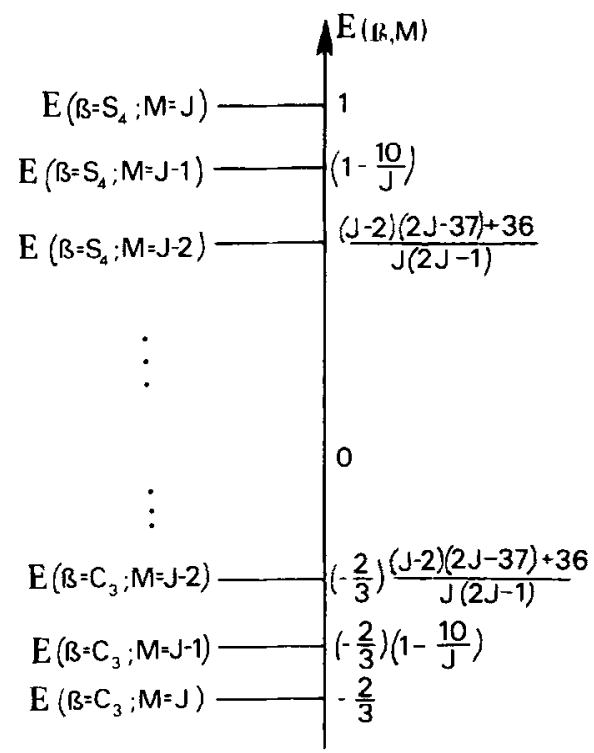

Fig. 2. Qualitative schema of the clusters in the spectra of octahedrally invariant fourth-rank tensor operators in the high- $J$ limit. 
TABLE I

Comparison (for Octahedrally Invariant Fourth-Rank Tensor Operators) of Relative Cluster Positions Predicted by First-Order Perturbation Theory and Centers of Gravity of Exactly Calculated Clusters

\begin{tabular}{|c|c|c|c|c|c|}
\hline J & B & $\begin{array}{l}E(J, B ; M=J) \\
E(J, E ; M=J-1)\end{array}$ & $\begin{array}{c}\text { Predicted } \\
\text { value }\end{array}$ & $\begin{array}{l}E(J, B ; M=J\} \\
E(J, B ; M=J-2)\end{array}$ & $\begin{array}{l}\text { Predicted } \\
\text { value }\end{array}$ \\
\hline 100 & $\mathrm{~S}_{4}$ & 1.111 & $10 / 9 \simeq$ & 1.2429 & \multirow{2}{*}{1.2429} \\
\hline 100 & $c_{3}$ & 1.109 & $=1.111$ & 1.2349 & \\
\hline 30 & $\mathrm{~S}_{4}$ & 1.4994 & \multirow{2}{*}{1.5} & 2.596 & \multirow{2}{*}{2.603} \\
\hline 30 & $c_{3}$ & 1.44 & & 2.16 & \\
\hline
\end{tabular}

\begin{tabular}{rrcc}
$\mathcal{J}$ & $M$ & $\begin{array}{c}E\left(J, B=C_{3} ; M\right) \\
E\left(J, B=S_{4} ; M\right)\end{array}$ & $\begin{array}{c}\text { predicted } \\
\text { value }\end{array}$ \\
\hline $\left.\begin{array}{rrr}100 & 100 & -0.667 \\
100 & 95 & -0.70 \\
30 & 30 & -0.67 \\
30 & 28 & -0.81\end{array}\right\}-2 / 3$ &
\end{tabular}

standard basis with $S_{4}$ quantization axis are smaller in the high- $J$ limit than the corresponding matrix elements in the basis oriented along the $C_{3}$ symmetry axis. The same fact is responsible for the difference in numbers for sixfold and eightfold degenerate clusters.

From formulas (6), (7), (10), (11), (13), and (14) one obtains immediately the relative positions of 6-fold, $E^{(k)}\left(M, C_{4}\right), 8$-fold, $E^{(k)}\left(M, C_{3}\right)$, and 12-fold, $E^{(k)}\left(M, C_{2}\right)$, degenerated clusters in the spectra of octahedrally invariant operators of orders 6 and 8:

$$
\begin{aligned}
& E^{(6)}\left(M, C_{4}\right): E^{(6)}\left(M, C_{3}\right): E^{(6)}\left(M, C_{2}\right)=1: \frac{16}{9}: \frac{-13}{8}, \\
& E^{(8)}\left(M, C_{4}\right): E^{(8)}\left(M, C_{3}\right): E^{(8)}\left(M, C_{2}\right)=1: \frac{8}{27}: \frac{9}{16} .
\end{aligned}
$$

One important consequence of the above statement permits qualitative prediction of the properties of the diagonal $F$ coefficients of Moret-Bailly $(7,8)$. These diagonal coefficients $F_{A_{1} p}^{(k, j)}$ are the diagonal matrix elements of a $k$-rank irreducible tensor operator calculated in the basis which diagonalizes the fourth-order tensor operator. Whenever standard basis functions with $M \approx J\left(\beta=C_{4}, C_{3}\right)$ are good approximations to the eigenfunctions of $T_{p}^{k}$ for any $k$ (in the high- $J$ limit) the eigenfunctions of $T_{A_{1}}^{(4)}$ corresponding to $M \approx J\left(\beta=C_{4}, C_{3}\right)$ are also the eigenfunctions of $T_{A_{1}}^{(k)}$ in good approximation. So the diagonal $F^{(k J n}$ coefficients must form 6-fold and 8-fold clusters as eigenvalues of $T_{A_{1}}^{k)}$ and $T_{A_{1}}^{(4)}$. Other types of clusters which may exist in the spectra of $T_{A_{1}}^{(k)}$ (for example, 12 -fold degenerate clusters 


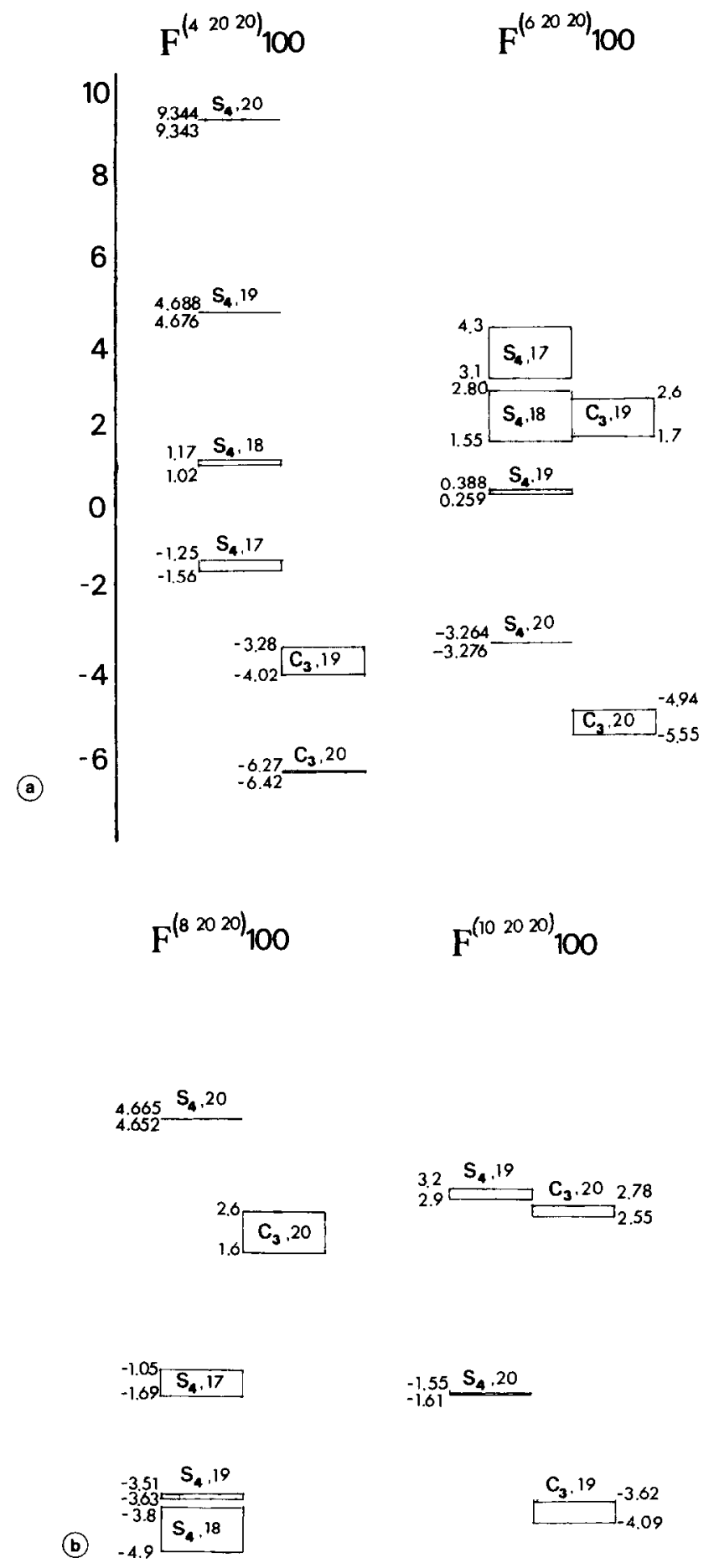

FIG. 3. Clusters of $F^{(k 20)}$ coefficients obtained by numerical calculations 
corresponding to $\beta=C_{2}$ axis) must not be present for $F^{(k J J)}$ coefficients. The absence of such clusters for $F^{(k J N}$ coefficients is due to the fact that the eigenfunctions of $T^{(k)}$ forming such clusters generate a subspace which in a good approximation coincides with the subspace of standard functions $\left|J, M\left(\beta=C_{2}\right)\right\rangle$, but the eigenfunctions of operator $T_{A 1}^{(4)}$ do not belong to this subspace, and to transform operator $T^{(k)}$ to the basis of eigenfunctions of $T_{A_{1}}^{(4)}$ we must utilize the orthogonal transformation which mixes functions corresponding to different clusters of eigenvalues of $T_{A_{1}}^{(k)}$. The relative positions of the clusters of $F^{(k J J)}$ coefficients may be calculated approximately by formulas (30)-(35). The scheme of cluster positions is more complicated for $k>4$ than for $k=4$ because the separation between clusters with different $M$ but the same $\beta$ is significantly larger. The scheme of clusters for $F^{(4 J J)}, F^{(6 J J)}, F^{(8 J J)}$, and $F^{(10 J J)}$ is shown in Fig. 3 for $J=20(8,21,22)$. Only the clusters which may be easily identified are shown. Each cluster is characterized by two numbers indicating the width of the cluster. The labeling of the clusters is the same as that for eigenfunctions of operator $T_{A_{1}}^{(4)}$. The relative positions of the clusters with different $M$ have a complicated behavior because $J$ is not large compared with the square of the tensor rank. Some numerical values of $F^{i k J n}$ are compared with predictions of simple perturbation theory in Tables II and III. The agreement between predicted values and the results of numerical calculations is worse for $F^{(k J J)}$ than for $F^{(4 J J)}$, which are identical with the eigenvalues of $T_{A_{1}}^{(4)}$. One of the reasons is insufficiently large $J$ values and the other is the differences between $F^{k J J}(k>4)$ coefficients and the eigenvalues of the operator $T_{A_{1}}^{(k)}$.

Until now only the relative positions of clusters were discussed. Two important questions arise in explaining the fine structure of each cluster. What is the symmetry of levels forming the clusters, and what is the order of levels in the cluster? To answer the first question it is sufficient to construct the induced representation of group $G$, starting from the one function $|\beta, M\rangle$ which was considered as a component of the irreducible representation of subgroup $G^{\prime} \subset G$ generated by axis $\beta$. This procedure was discussed earlier $(5,9)$ and need not be repeated here. The calculation of the level ordering in each cluster is a more complicated problem. The result depends on the type of tensor operator. The theory has already been formulated for an octahedrally invariant fourth-rank tensor operator (5).

TABLE II

Comparison of Relative Positions of Different Types Clusters of $F^{(6 J)}$ Coefficients with the Predicted Value for Clusters in the Spectrum of the $T_{A r}^{6:}$ Operator

\begin{tabular}{|c|c|c|c|}
\hline \multirow{2}{*}{ J } & \multirow{2}{*}{ M } & 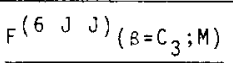 & \multirow{2}{*}{$\begin{array}{l}\text { Predicted } \\
\text { value }\end{array}$} \\
\hline & & $\left.F^{(6} \mathrm{J} J\right)\left(B=S_{4} ; M\right)$ & \\
\hline 100 & 100 & 0.564 & \\
\hline 100 & 96 & 0.83 & \\
\hline 30 & 30 & 0.587 & $9 / 16=$ \\
\hline 20 & 20 & 0.63 & $=0.5625$ \\
\hline
\end{tabular}


TABLE III

Relative Positions of Clusters for Different Diagonal $F^{(k J)}$ Coefficients $^{2}$

\begin{tabular}{|c|c|c|c|c|c|c|c|}
\hline $\mathrm{J}$ & $B$ & $\frac{F^{(6 \mathrm{~J} J)}(\beta ; M=J)}{F^{(6 J J)}\langle(B ; M=J-1)}$ & $\begin{array}{l}\text { Predicted } \\
\text { value }\end{array}$ & $\frac{\left.\left.F^{(8}\right\lrcorner J\right)_{(\beta ; M=J)}}{F^{(8 J J)_{(B ; M=J-1)}}}$ & $\begin{array}{c}\text { Predicted } \\
\text { value }\end{array}$ & $\frac{F^{\{10\lrcorner J)}{ }_{(\beta ; M=J)}}{F^{(10 J J)}(\beta ; M=J-1)}$ & $\begin{array}{l}\text { Predicted } \\
\text { value }\end{array}$ \\
\hline 100 & $s_{4}$ & 1.2661 & $100 / 79 \approx$ & & $25 / 16=$ & & $20 / 9=$ \\
\hline 100 & $c_{3}$ & 1.281 & $f=1.2658$ & & $f=1.5625$ & & $f=2.2$ \\
\hline 20 & $\mathrm{~s}_{4}$ & -8.4 & \multirow{2}{*}{-20} & -1.30 & \multirow{2}{*}{-1.25} & -0.52 & \multirow{2}{*}{$\begin{array}{l}-4 / 7= \\
=0.57\end{array}$} \\
\hline 20 & $c_{3}$ & -2.6 & & $\begin{array}{c}\text { not } \\
\text { defined }\end{array}$ & & -0.7 & \\
\hline
\end{tabular}

${ }^{a}$ Predicted values are calculated by formulas (31).

Extension to other tensor operators and $F$ coefficients is also possible, but we do not treat it here. Instead we now focus attention on clusters in the spectra of nondiagonal tensor operators.

\section{NONDIAGONAL TENSOR OPERATORS}

\section{A. Introductory Calculations}

Let us now study the energy-level splitting caused by nondiagonal tensor operators. The simplest Hamiltonian has the form

$$
H=B T^{(0)}+\lambda T^{(k)},
$$

where $T^{(0)}$ is a zero-order tensor operator and $T^{(k)}$ is a tensor operator of order $k$ which is assumed to be completely nondiagonal in $J$ ( $J$ is the quantum number which distinguishes the nondegenerate levels of the zero-order operator). We now investigate the case of weak coupling. This means that the perturbation theory which treats $T^{k\rangle}$ as a perturbation is applicable. Only second-order perturbation theory gives nonzero splitting. Before formulating a general approach, we consider one concrete example to demonstrate the existence of clusters in the weak coupling limit for nondiagonal tensor operators. One of the most physically interesting problems is the splitting caused by a tetrahedrally invariant tensor operator of third order (see Section IV). The same operator is important for the calculation of the vibration-rotation band intensities in the spectra of tetrahedral molecules (26). So the Hamiltonian is

$$
H=B T_{A_{1}}^{(0)}+V_{A_{1}}^{(3 u)},
$$

and $B$ is assumed to be sufficiently large that second-order perturbation theory is applicable. The symmetry-adapted eigenfunctions of the zero-order operator are denoted by $\left|J_{\alpha} n, C, \sigma\right\rangle$, where the additional index $\alpha$ is introduced to simplify the relation between $T_{d}$ and $O(3)$. (For $J$ even, $\alpha=g$ and for $J$ odd, $\alpha=u$.) $n$ is now the internal multiplicity index which distinguishes the different eigenvalues of fourth-order diagonal tensor operators. 
The second-order correction to the energy level may be easily written in the basis mentioned above,

$$
\begin{aligned}
E^{(2)}\left(J_{\alpha} ; n, C\right)= & -\frac{1}{2 B(J+1)} \sum_{n^{\prime}}\left|\left\langle J_{\alpha} ; n, C, \sigma\left|V_{A_{1}}^{(3 u)}\right| J_{\alpha}+1 ; n^{\prime}, C, \sigma\right\rangle\right|^{2} \\
& -\frac{1}{6 B(J+2)} \sum_{n^{\prime}}\left|\left\langle J_{\alpha} ; n, C, \sigma\left|V_{A_{1}}^{(3 u)}\right| J_{\alpha}+3 ; n^{\prime}, C, \sigma\right\rangle\right|^{2} \\
& +\frac{1}{2 B J} \sum_{n^{\prime}}\left|\left\langle J_{\alpha} ; n, C, \sigma\left|V_{A_{1}}^{(3 u)}\right| J_{\alpha}-1 ; n^{\prime}, C, \sigma\right\rangle\right|^{2} \\
& +\frac{1}{6 B(J-1)} \sum_{n^{\prime}}\left|\left\langle J_{\alpha} ; n, C, \sigma\left|V_{A_{1}}^{(3 u)}\right| J_{\alpha}-3 ; n^{\prime}, C, \sigma\right\rangle\right|^{2} .
\end{aligned}
$$

The matrix elements of $V_{A_{1}}^{(3 u)}$ are expressed in terms of the

coefficients

$$
\left.F_{A 1 p}^{(3, j} J_{u} J_{g}\right)
$$

$$
\begin{aligned}
& \left\langle J_{g}, p\left|V_{A 1}^{(3 u)}\right| J_{u}^{\prime}, p^{\prime}\right\rangle=F_{A_{1} p}^{\left(33_{k} J_{g}^{\prime} J^{\prime \prime}\right)}(-1)^{\left(J_{g}+J_{u}^{\prime}+3\right) / 2} \\
& \times\left[\frac{7\left(2 J_{g}+1\right)\left(2 J_{u}+1\right)}{4}\right]^{1 / 2}\left(\begin{array}{ccc}
J_{g} & 3 & J_{u} \\
0 & 0 & 0
\end{array}\right) .
\end{aligned}
$$

Using Eq. (41), one may simplify Eq. (40) to

$$
\begin{aligned}
& E^{(2)}\left(J_{\alpha} ; n, C\right)=a_{J+1} \sum_{n^{\prime}}\left|F_{A_{1} n C n^{\prime} C}^{\left(3_{u} J_{\alpha} J_{\alpha}+1\right)}\right|^{2}+a_{J+3} \sum_{n^{\prime}}\left|F_{A_{1} n C n^{\prime} C}^{\left(3_{u} J_{\alpha} J J_{\alpha}+3\right)}\right|^{2} \\
& +a_{J-1} \sum_{n^{\prime}}\left|F_{A 1 n C n^{\prime} C}^{\left(3_{u} J_{\alpha} J_{\alpha}-1\right)}\right|^{2}+a_{J-3} \sum_{n^{\prime}}\left|F_{A_{1} n C n^{\prime} C}^{\left(3_{u} J_{\alpha} J_{\alpha}-3\right)}\right|^{2}
\end{aligned}
$$

where

$$
\begin{aligned}
& a_{J+1}=-\frac{21}{16 \pi B} \frac{J(J+2)}{(2 J-1)(2 J+5)} \underset{J \rightarrow \infty}{\rightarrow}-\frac{21}{64 \pi B}, \\
& a_{J-1}=\frac{21}{16 \pi B} \frac{(J-1)(J+1)}{(2 J-3)(2 J+3)} \underset{J \rightarrow \infty}{\rightarrow} \frac{21}{64 \pi B}, \\
& a_{J+3}=-\frac{35}{48 \pi B} \frac{(J+1)(J+3)}{(2 J+3)(2 J+5)} \underset{J \rightarrow \infty}{\rightarrow}-\frac{35}{192 \pi B}, \\
& a_{J-3}=\frac{35}{48 \pi B} \frac{J(J-2)}{(2 J-1)(2 J-3)} \underset{J \rightarrow \infty}{\rightarrow} \frac{35}{192 \pi B} .
\end{aligned}
$$

If one prefers to use $F$ symbols for group $S O(3)$ but not $O(3)(24)$, then two separate equations for $J$, even and odd, are to be used:

$$
\begin{aligned}
& E^{(2)}\left(I_{g} ; n, C\right)=a_{J+1} \sum_{n^{\prime}}\left|F_{A_{2} n C n^{\prime} C^{\prime}}^{\left(3 J_{g}+1\right)}\right|^{2}+a_{J+3} \sum_{n^{\prime}}\left|F_{A_{2} n C n^{\prime} C}^{\left(3 J_{g} J_{g}+3\right)}\right|^{2} \\
& +a_{J-1} \sum_{n^{\prime}}\left|F_{A_{2} n C n^{\prime} \bar{C}}^{\left(3 J_{g} J_{g}-1\right)}\right|^{2}+a_{J-3} \sum_{n^{\prime}}\left|F_{A_{2} n C n^{\prime} \bar{C}}^{\left(3 J_{g} J_{g}-3\right)}\right|^{2}
\end{aligned}
$$




\section{TABLE IV}

Comparison of the Clustering in the Spectra of $T_{A_{1}}^{(40)}$ and $T_{A_{1}}^{(3 u)}$ Operators for $J=16$

\begin{tabular}{|c|c|c|c|c|c|}
\hline $\mathrm{nt}$ & $c$ & $E^{(2)}\left(T_{A_{1}}^{(3 u)} ; J=16 ; n, C\right) 10^{4}$ & 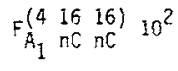 & $n$ & $c$ \\
\hline 1 & $A_{1}$ & 19.12 & 9.821 & 1 & $A_{1}$ \\
\hline 3 & $F_{1}^{*}$ & $19.00\} 6$ & \begin{tabular}{l|l}
6 & 9.815
\end{tabular} & 3 & $F_{1}$ \\
\hline 2 & $E$ & 18.93 & 19.812 & 2 & $E^{2}$ \\
\hline 3 & $F_{2}$ & $12.31 / 6$ & $6\left\{\begin{array}{l}3.75 \\
0\end{array}\right.$ & 3 & $F_{2}$ \\
\hline \multirow[t]{2}{*}{2} & $F_{1}$ & $10.85\}^{\circ}$ & $\{3.66$ & 2 & $F_{1}$ \\
\hline & & & $-0.3 n$ & 1 & E \\
\hline 1 & $\mathrm{E}$ & 7.46 & $6-0.51$ & 2 & $\mathrm{~F}_{2}$ \\
\hline 2 & $\mathrm{~F}_{2}$ & 5.46 & -1.10 & 0 & $A_{2}$ \\
\hline 1 & $F_{2}$ & $1^{-0.61}$ & -2.98 & 1 & $\mathrm{~F}_{2}$ \\
\hline 0 & $A_{2}$ & \begin{tabular}{l|l}
8 & -1.99
\end{tabular} & -3.55 & 1 & $F_{1}$ \\
\hline 1 & $F_{1}$ & $\{-2.99$ & -4.17 & 0 & $A_{1}$ \\
\hline 0 & $A_{1}$ & -3.24 & & & \\
\hline 0 & $\mathrm{~F}_{2}$ & $j^{-24.51}$ & $-6.55 \mid$ & 0 & $F_{1}$ \\
\hline 0 & $E^{c}$ & $8)-24.84$ & 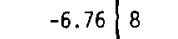 & 0 & E \\
\hline 0 & $F_{1}$ & -25.28 & -6.98 & 0 & $F_{2}$ \\
\hline
\end{tabular}

$$
\begin{array}{rl}
E^{(2)}\left(J_{u} ; n, C\right)=a_{J+1} \sum_{n^{\prime}}\left|F_{A_{2} n \bar{C} n^{\prime} C}^{\left(3 J_{u} J_{u}+1\right)}\right|^{2}+a_{J+3} \sum_{n^{\prime}}\left|F_{A z}^{\left(3 J_{u} J_{u}^{u}+3\right)}\right|^{2} & 2 \\
& +a_{J-1} \sum_{n^{\prime}}\left|F_{A_{2} n \bar{C} n^{\prime} \bar{C}}^{\left(3 J_{u} J^{\prime}-1\right)}\right|^{2}+a_{J-3} \sum_{n^{\prime}}\left|F_{A_{2} n \bar{C} n^{\prime} C}^{\left(3 J_{u} J_{u}-3\right)}\right|^{2} .
\end{array}
$$

Here $\bar{C}=\left\{A_{2}\right.$ if $C=A_{1} ; A_{1}$ if $C=A_{2} ; E^{*}$ if $C=E ; F_{2}$ if $C=F_{1} ; F_{1}$ if $\left.C=F_{2}\right\}$. More precisely for doubly degenerate representation we have the correspondence $E 1 \leftrightarrow E 2$ which follows from the special choice of the matrix representation (24).

Equations (44) and (45) give the solution of the problem considered through the second-order perturbation theory. Factor $7 / 16 \pi B$ may be eliminated from expressions (44) and (45): The resulting numerical values of $E^{(2)}(J ; n, C)$, calculated with the $F^{3}$ coefficients from Hilico's tables $(25,26)$, are listed in Table IV for $J=16$. For comparison the eigenvalues of a diagonal fourth-rank operator are presented in the same table. Even for such a relatively low $J$ value $(J=16)$ the clustering of eigenvalues exists for the third-order nondiagonal tensor operator. The levels which form the clusters are denoted by curly brackets, with the numbers corresponding to the quasi degeneracy of the clusters. One can see that the relative numbers of sixfold and eightfold degenerate clusters are different for third- and fourth-order operators. This fact will be discussed in the following subsection. Here, more detailed results are presented using tetrahedrally invariant third-rank tensor operators as an example.

It is well known that any tensor operator may be represented as a sum of equivalent operators ${ }^{(\Delta J=s)} T^{(k)}$,

$$
T^{(k)}=\sum_{s}^{(\Delta J=s)} T^{(k)},
$$


TABLE V

Spectra of Equivalent Nondiagonal Tensor Operators ${ }^{[\Delta J} T_{A 1}^{3 u)}$ Computed by Second-Order Perturbation Theory for $J=16$

\begin{tabular}{|c|c|c|c|c|}
\hline$n C$ & $|+3|_{V}\{3\rangle$ & $|-3|_{y^{(3)}}$ & $|-3|_{V}{ }^{(3)}+$ & $|+3|_{V}(3)$ \\
\hline $1 A_{1}$ & -8.414 & 0.014 & -8.400 & \multirow{3}{*}{6} \\
\hline $3 F_{1}^{1}$ & $-8.416 \quad 6$ & 0.008 & -8.407 & \\
\hline $2 E^{1}$ & $-8.416)$ & 0.005 & -8.411 & \\
\hline $3 F_{2}$ & $-14.536 / 6$ & $11.847 / 6$ & -2.689 & \multirow{2}{*}{\}$^{6}$} \\
\hline $2 F_{1}^{2}$ & -14.573 & $11.825\}^{\circ}$ & -2.748 & \\
\hline $1 E$ & -18.627 & 19.735 & 2.108 & \multirow{3}{*}{6} \\
\hline $2 \mathrm{~F}_{2}$ & -18.761 & 19.872 & 1.111 & \\
\hline $\mathrm{OA}_{2}$ & -19.077 & 20.016 & 0.939 & \\
\hline $1 F_{2}$ & -21.124 & 24.214 & 3.089 & \multirow[t]{2}{*}{6} \\
\hline $1 F_{1}^{2}$ & -21.628 & 25.055 & 3.439 & \\
\hline $0 A_{1}$ & -22.274 & 26.348 & \multicolumn{2}{|l|}{4.073} \\
\hline$O F_{1}$ & -23.874 & 28.116 & \multicolumn{2}{|l|}{4.241} \\
\hline$O E$ & -24.114 & 28.617 & \multicolumn{2}{|l|}{4.504} \\
\hline $\mathrm{OF}_{2}$ & -24.342 & 29.098 & \multicolumn{2}{|l|}{4.756} \\
\hline
\end{tabular}

\begin{tabular}{|c|c|c|c|c|c|c|c|}
\hline$n c$ & {$[+1]_{V}(3)$} & $n c$ & {$[-1]_{V}^{(3)}$} & & $n C$ & {$\left[+1 V_{V}^{(3)}+\right.$} & 1] $V^{(3)}$ \\
\hline $2 \mathrm{E}$ & -0.624 & $1 \mathrm{E}$ & 53.468 & & $1 A_{1}$ & 27.524 & \\
\hline $3 F_{1}$ & $-0.710 \quad 6$ & $2 \Gamma_{2}$ & 49.440 & & $3 F_{1}$ & 27.406 & 6 \\
\hline $1 A_{1}$ & -0.880 & $1 F_{2}$ & 47.298 & & $2 E^{2}$ & 27.346 & \\
\hline $2 F_{1}$ & \begin{tabular}{l|l}
-27.004 & 6
\end{tabular} & $0 A_{1}$ & 47.002 & & $3 F_{2}$ & 14.997 & 6 \\
\hline $3 F_{2}$ & $-28.851\}^{\circ}$ & $1 F_{1}$ & 44.265 & & $2 F_{1}$ & 13.603 & \\
\hline $\begin{array}{ll}0 F_{1} \\
0 F\end{array}$ & -33.057 & $\begin{array}{l}3 F_{2} \\
2 F_{1}\end{array}$ & $\begin{array}{l}43.848 \\
40.607\end{array}$ & & $1 E$ & 6.351 & \\
\hline $\begin{array}{l}0 \mathrm{E} \\
0 \mathrm{~A}_{2}\end{array}$ & $\begin{array}{l}-34.882 \\
-36.437\end{array}$ & 1 & 33.512 & & $2 F_{2}$ & 4.347 & \\
\hline $\begin{array}{lll} & A_{2} \\
0 & F_{2}\end{array}$ & $\begin{array}{l}-36.437 \\
-36.765\end{array}$ & $1 A_{1}^{2}$ & 28.404 & & $\mathrm{OA}_{2}$ & -2.925 & \\
\hline $2 \mathrm{~F}_{\hat{z}}$ & -45.093 & $3 F_{1}$ & 28.116 & 6 & $1 \mathrm{~F}_{2}$ & -3.698 & 8 \\
\hline $1 \mathrm{E}^{\mathrm{C}}$ & -47.117 & $2 E$ & 27.970 & & $\begin{array}{ll}1 & F_{1} \\
0 & A_{1}\end{array}$ & $\begin{array}{l}-5.432 \\
-7.319\end{array}$ & \\
\hline $1 F_{1}$ & -50.697 & & 7.498 & & $O F$, & -29.552 & \\
\hline $1 F_{2}$ & -50.996 & $O E^{2}$ & 5.533 & 8 & $0 \mathrm{E}^{1}$ & $\begin{array}{l}-29.532 \\
-29.348\end{array}$ & 8 \\
\hline $0 \mathrm{~A}_{1}$ & -54.321 & $0 F_{1}$ & 3.534 & & $\mathrm{OF}_{2}$ & -29.267 & \\
\hline
\end{tabular}

such that ${ }^{(\Delta J=s)} T^{k)}$ has nonzero matrix elements only between the states with $\Delta J=s(17)$. The operator $T_{A_{1}}^{3 u)}$ considered in this section may be expressed as

$$
T_{A_{1}}^{3 u)}={ }^{(\Delta J=-3)} T_{A_{1}}^{(3 u)}+{ }^{(\Delta J=-1)} T_{A_{1}}^{(3 u)}+{ }^{(\Delta J=1)} T_{A_{1}}^{(3 u)}+{ }^{(\Delta J=3)} T_{A_{1}}^{3 u)} .
$$

All other equivalent tensor operators are identically zero. The question is: What are the specific features of the spectra of each equivalent nondiagonal tensor operator? The numerical answer for $J=16$ is presented in Table V, where the spectra of operators ${ }^{(\Delta J= \pm 3)} T_{A 1}^{3 u)}$ and ${ }^{(\Delta J- \pm 1)} T_{A_{1}}^{(3 u)}$ and their linear combinations are 
listed. Some clusters are presented in each spectrum, but the types and numbers of well-pronounced clusters vary. The final clustering presented in Table IV for the total $T_{A_{1}}^{(3 u)}$ tensor operator is obtained by near cancellation of many important terms. Although the qualitative structure of the $T_{A_{1}}^{(3 u)}$ total operator spectrum seems to be rather simple, the spectra of equivalent nondiagonal tensor operators are different.

One note more before general consideration of the nondiagonal tensor operators. The clustering in the spectrum of operator $T_{A_{1}}^{3 u)}$ is connected with clustering of $F^{\left(3 J J^{\prime}\right)}$ coefficients. This phenomenon was given little attention (although some regularities in the $F^{(3 J J \pm 3)}$ coefficients were marked $(11,26)$ and approximate formulas for their calculations were derived), probably because the $F^{\left(3 J J^{\prime \prime}\right)}$ do not themselves form well-defined clusters, but specific linear combinations of their squares which arise naturally in the perturbation expressions. For $F^{(3 J J \pm 3)}$ coefficients these linear combinations may be simplified considerably because of the well-known fact that only one of the $F_{A_{1}}^{\left(3, J C^{\prime} n^{\prime} C\right.}\left(n, n^{\prime}=0,1,2, \ldots\right)$ coefficients is essential (26), so for the equivalent nondiagonal tensor operator ${ }^{(\Delta J=3)} T_{A_{1}}^{(3 u)}$ the second-order energy contribution is to good approximation due to only one $F$ coefficient. In such a case, one may force clustering by an appropriate choice of the phases for the $G$ coefficients, which was made arbitrarily (8). Nevertheless, only the squares of the moduli of coefficients nondiagonal in $J$ are physically meaningful.

\section{B. Qualitative Discussion}

To explain qualitatively the structure of nondiagonal tensor operator spectra consider again the commutativity relations between $T_{p}^{k)}$ and $J_{0}$. The commutator is nondiagonal in $J$, so the matrix elements of type

$$
\left\langle J^{\prime} M+\mu\left|T_{\mu}^{(k)}\right| J M\right\rangle
$$

are to be calculated. First, we estimate the asymptotics of these matrix elements in the high- $J$ limit for a $T_{A_{1}}^{(3 u)}$ tetrahedrally invariant tensor operator. Two particular forms of this operator are considered: those given by formulas (4) and (8). The matrix representation of the commutator is shown schematically in Figs. 4 and 5. The main difference from Fig. 1 is the nondiagonal-in- $J$ character of these matrices. So the good commutativity relation between $T_{A_{1}}^{(3 u)}$ and $J_{0}$ may be achieved only in the small coupling limit, when the difference between zero-order levels is significantly larger than the splitting of each level. If this is the case then to yield the same cluster structure for nondiagonal operator as for diagonal one matrix elements,

$$
\left\langle J^{\prime} J+\mu\left|T_{\mu}^{(k)}\right| J J\right\rangle
$$

must be significantly smaller in the high- $J$ limit than

$$
\left\langle J^{\prime} \mu\left|T_{\mu}^{(k)}\right| J 0\right\rangle \text {. }
$$

In this respect the two forms of $T_{A_{1}}^{(3 u)}$, (4) and (8), have different properties. If operator $T_{A_{1}}^{(3 u)}$ is written in the standard basis with the $S_{4}$ symmetry axis as the axis of quantization, then the matrix elements (50) are constants, whereas (49) 


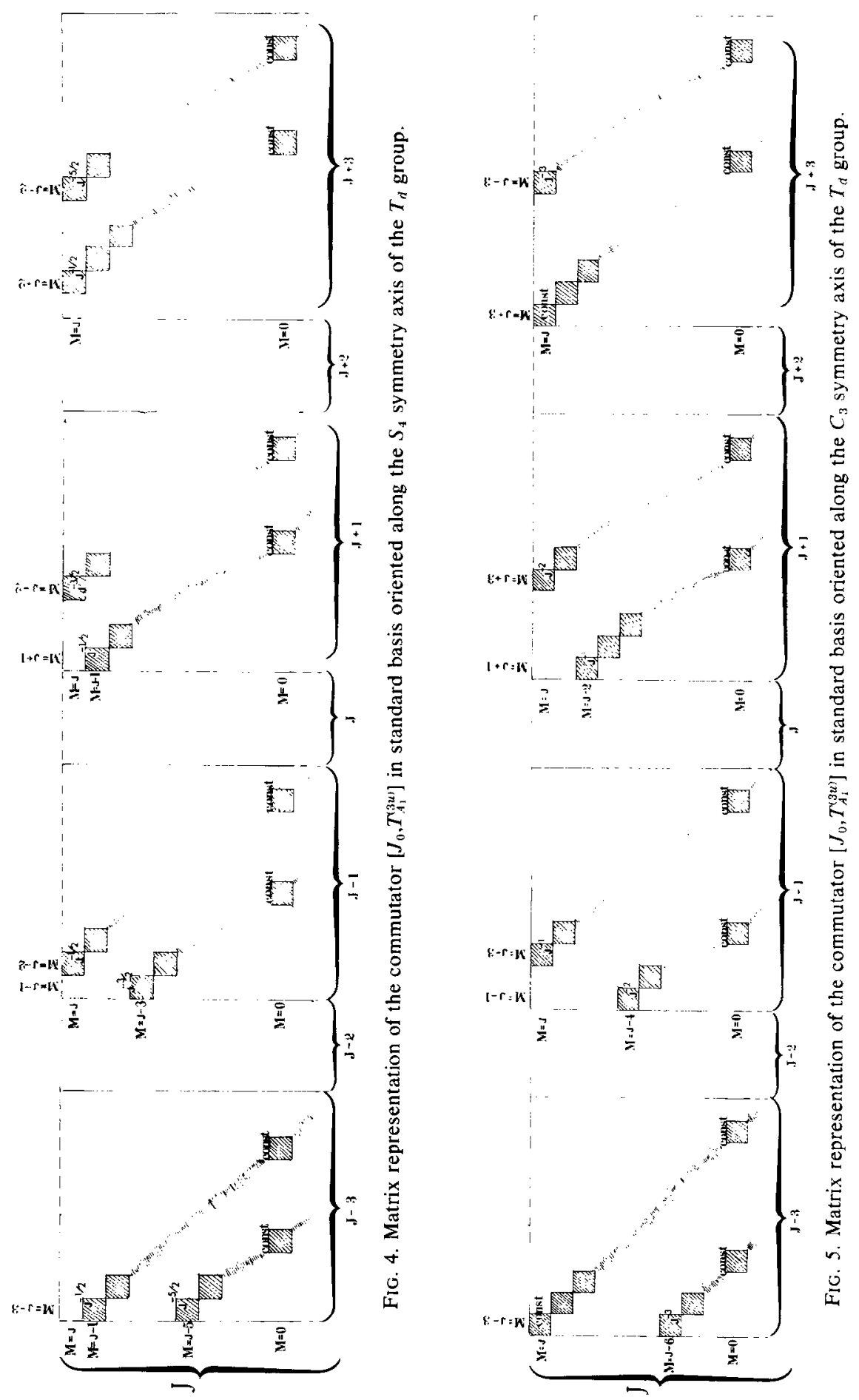


goes to zero as $J$ increases. In contrast, for $T_{A_{1}}^{(3 u)}$ written in the standard basis with the $C_{3}$ symmetry axis as the axis of quantization, both types of matrix elements, (49) and (50), are constants in the high- $J$ limit. The same arguments that were applied to discussion of diagonal tensor operator spectra now lead us to conclude that sixfold degenerate clusters must be significantly more pronounced than eightfold degenerate clusters. Tables IV and V verify this conclusion. To predict the cluster structure in nondiagonal tensor operator spectra the general matrix element asymptotics are to be taken into account:

$$
\left\langle J^{\prime} J+\mu\left|T_{\mu}^{(k)}\right| J J\right\rangle \underset{J \rightarrow \infty}{\rightarrow} c(J)^{\left(J^{\prime}-J-\mu\right) / 2},
$$

where $c$ is a numerical constant. Formula (51) shows that the most unfavorable case for clustering in the spectra of nondiagonal tensor operators is case where the operator $T_{p}^{(k)}$ includes the standard basis component $T^{(k)}$ with $\mu=\left(J^{\prime}-J\right)$. In such a case the asymptotics for matrix elements with $M \approx J$ are approximately the same as for $M \approx 0$, and equal constants. So the subspace with "good commutativity" between $J_{0}$ and $T_{p}^{(k)}$ cannot be extracted.

Approximate calculations of cluster positions may be done for nondiagonal operators using zero-order standard basis functions in analogy with the procedure of Section IIB, but the calculation is more tedious in this case and does not result in simple formulas as for the diagonal operators. So it will not be discussed here.

\section{Strong Coupling Case}

It has already been noted that the formation of clusters in the spectra of the nondiagonal tensor operators discussed above takes place only in the weak coupling limit. We now discuss briefly the possible alternative case of strong coupling. From the physical point of view the strong coupling case corresponds to infinitely large constant $\lambda$ in Eqs. (1) and (38), but it is not possible to omit the zero-order tensor operator to reach the approximate operator suitable for the strong interaction limit. If the weak coupling limit describes the small perturbations of a free rigid rotator by a "crystal field," then strong coupling corresponds to the motion of the particle in one of the minima of the "crystal potential." In the zero-order approximation, each of the crystal potential minima is treated independently. So in the strong coupling limit degenerate levels exist which form clusters, and the origin of these clusters is the existence of several equivalent minima of the crystal-field potential (27). The possibility of tunneling from one minimum to another leads to the splitting of degenerate levels. To illustrate here the general idea of cluster formation in the case of the strong coupling limit, we consider the particular example of the spectra of operator (39) under the assumption of strong interaction (28). The crystal-field potential $V_{A_{1}}^{(3 u)}$ possesses four minima corresponding to the particle positions above four equivalent sides of a tetrahedron. The most crude approximation is to suppose that the motion of the particle in each independent minimum is described by a two-dimensional harmonic oscillator Hamiltonian. To improve this model it is necessary to break the symmetry from the $S U(2)$ group to $S O(2)$ and to $C_{3 v}$ : This gives the level structure for one independent minimum. To take into account the existence of four equivalent 
TABLE VI

Subduction/Induction Table for Constructing Energy-Level Clusters for Tetrahedrally Invariant Third-Rank Tensor Operators in the Strong Coupling Case

\begin{tabular}{ccccc}
\hline$S U(2)$ & 0 & 1 & 2 & 3 \\
\hline$S 0(2)$ & 0 & 1 & 0,2 & 1,3 \\
\hline$C_{3 V}$ & $A_{1}$ & $E$ & $A_{1}, E$ & $E, A_{1}+A_{2}$ \\
\hline$T_{d}$ & $A_{1}+F_{2}$ & $E+F_{1}+F_{2}$ & $\begin{array}{c}A_{1}+F_{2} \\
+F_{1}+F_{2}\end{array}$ & $\begin{array}{c}E+F_{1}+F_{2} \\
\left(A_{1}+F_{2}\right)+\left(A_{2}+F_{1}\right)\end{array}$ \\
\hline
\end{tabular}

minima one need construct the induced representation of $T_{d}$ starting from the irreducible representation of $C_{3 v}$. Thus each level generates a cluster of four or eight levels depending on degeneracy. The procedure described above yields the level structure presented in Table VI for some low vibrational levels. The strong coupling limit is only sketched here, because the aim is to demonstrate that clusters of four and eight levels arise naturally in the strong-field model for tetrahedrally invariant third-rank tensor operators, whereas six- and eightfold clusters are formed in the small coupling case. Moreover, the sixfold clusters are more pronounced. The correlation between strong and weak coupling limits must explain this behavior.

\section{PHYSICAL APPLICATIONS}

In this section we discuss some physical problems for which the method developed earlier is applicable. The most widely known problem is the centrifugal distortion of spherical tops $(3-10)$. The corresponding rotation Hamiltonian for the ground state has the form

$$
H=H_{A_{1}}^{(0 g)}+H_{A_{1}}^{(q g)}+H_{A_{1}}^{(6 g)}+H_{A_{1}}^{(g g)}+\cdots .
$$

The $H_{A_{1}}^{(40)}$ term is usually the most important, and higher-order tensor operators yield small corrections. It is well known that the spectrum of a fourth-order tensor operator possesses remarkable clustering in the high- $J$ limit. The interesting question is whether or not the inclusion of higher-order terms in the energy expression violates such clustering from the qualitative point of view. The reasoning developed in this paper shows that clustering of energy levels exists for operator (52) in the high- $J$ limit for any form of operator. The only limitation is the diagonal character of this operator (the case of weak interaction may be treated identically). The rotation Hamiltonian is always $J$ diagonal. So the clustering of rotational energy levels for spherical tops in the high- $J$ limit is a general phenomenon. If operator (52) includes some important terms of high order, the same clusters appear as for a fourth-order operator, but the relative positions of the clusters change significantly. Thus, with the cluster structure of the experimental spectra known, it is possible to construct the linear combination of tensor operators resulting in such spectra. Formulas (30)-(35) are key formulas for this interpretation.

The other physical problem is the large-amplitude vibrations of inorganic non- 
rigid ionic molecules composed of a highly symmetrical rigid core and an atom which possesses almost free motion around this core. The appropriate examples are $\mathrm{LiBH}_{4}, \mathrm{~T} 1 \mathrm{ReO}_{4}, \mathrm{~T}_{1} \mathrm{NO}_{3}$, etc. There exist some nonempirical calculations of the potential energy surfaces for such molecules (29) and a number of electron diffraction data $(14,30)$. The low-frequency spectroscopic investigations of such molecules in the gas phase are rather complicated. So to predict the properties of these molecules in gas phase under high-temperature conditions theoretical models are necessary. Such investigations were begun recently by the author of the present article and his collaborators $(15,16,28,31,33)$. To relate the general treatment of the problem considered in this article to specific nonrigid molecules, we consider $\mathrm{LiBH}_{4}$ as an example. Nonempirical study of this molecule (29) shows that the $\mathrm{Li}$ atom possesses a large-amplitude motion about the $\mathrm{BH}_{4}$ rigid core. The potential energy minima correspond to the $\mathrm{Li}$ position above the middle of the side of the rigid tetrahedron ( $C_{3 v}$ symmetry), whereas the Li position above the top of the tetrahedron corresponds to a maximum of potential energy. The potential energy barrier separating one minimum from another corresponds to the $C_{2 v}$ position of the $\mathrm{Li}$ atom, and it is sufficiently low to permit the tunneling of $\mathrm{Li}$ from one minimum to another at reasonable temperatures. This is the qualitative description of $\mathrm{LiBH}_{4}$ which is probably more suited for other examples of similar molecules. $\mathrm{LiBH}_{4}$ is a natural starting point because of the existence of nonempirical calculations which are surely not good enough to calculate the vibrationrotation energy levels but which nevertheless are qualitatively sufficient for constructing simple models of vibration-rotation spectra. The most crude approximation is to fix the $\mathrm{BH}_{4}$ core as a rigid tetrahedron and to consider $\mathrm{Li}$ as a rigid rotator in the tetrahedrally invariant field of the rigid core (33). The simplest form of potential reproducing the regularities described above is the $V_{A_{1}}^{(3 u)}$ tensor operator which is tetrahedrally but not octahedrally invariant. $V_{A_{1}}^{(3 u)}$ possesses only four minima and it is antisymmetrical with respect to inversion. Thus the most simple model for treating the large-amplitude deformation of $\mathrm{LiBH}_{4}$ is that described by Hamiltonian (39) studied in Section III.

\section{CONCLUSION}

An attempt to explain the general phenomenon of energy-level clustering in the spectra of tensor operators adapted to a nonstandard basis was undertaken in this article. A mathematical treatment of tensor operators was developed which is formally equivalent to the intuitive assumptions of basis functions by Harter and Patterson (5). Tests involving the commutativity of $J_{0}$ and $T_{p}^{(k)}$ were proposed which indicate the types of basis functions to be used for clustered states without reference to the corresponding potential surface. Although a number of important questions are still unresolved the clustering itself no longer appears to be mysterious or unexpected. The most interesting problem to be solved, in the author's opinion, is the correlation between weak and strong coupling limits for nondiagonal tensor operators. It seems also that a more rigorous mathematical explanation of tensor operator spectra is possible using the permutation symmetry associated with a given tensor operator. From the intuitive, physical point of view such symmetry is due to permutation symmetry of the localized standard basis functions. 
Additional numerical examples are also desirable, especially for tensor operators adapted to some different chains of groups, $G \subset O(3)$.

\section{ACKNOWLEDGMENTS}

The author wants to thank the Laboratoire de Spectronomie Moléculaire de l'Université de Dijon for hospitality during his stay in France. Valuable comments of and discussions with J. Moret-Bailly, H. Berger, J. C. Hilico, and F. Michelot are gratefully acknowledged. Many thanks are due Dr. W. M. Gelbart for improving the manuscript. The author is indebted to the referee of this Journal for many corrections and constructive criticism.

ReCEIVed: April 7, 1978

\section{REFERENCES}

1. J. P. Aldridge, H. Filip, H. Fliker, R. F. Holland, R. S. McDowell, N. G. Nereson, and K. Fox, $J$. Mol. Spectrosc. 58, 165-168 (1975).

2. R. S. McDowell, H. W. Galbraith, B. J. Krohn, C. D. Cantrell, and E. D. Hinkley, Opt. Commun. 17, 178-181 (1976).

3. K. Fox, H. W. Galbraith, B. J. Krohn, and J. D. Louck, Phys. Rev. 15, 1363-1381 (1977).

4. B. J. KROHN, J. Mol. Spectrosc. 68, 497-498 (1977).

5. W. G. Harter and C. W. Patterson, J. Chem. Phys. 66, 4872-4885 (1977); C. W. Patterson and W. G. Harter, J. Chem. Phys. 66, 4886-4892 (1977); W. G. Harter, C. W. Patterson, and F. J. DaPaiXao, Rev. Mod. Phys. 50, 37 (1978); W. G. Harter and C. W. Patterson, to be published.

6. K. T. Неснт, J. Mol. Spectrosc. 5, 355-389 (1960).

7. J. Moret-Bailly, Cah. Phys. 15, 237-314 (1961).

8. J. Moret-Bailly, L. Gautier, and J. Montagutelli, J. Mol. Spectrosc. 15, $355-377$ (1965).

9. A. J. Dorney and J. K. G. Watson, J. Mol. Spectrosc. 42, 135-148 (1972).

10. S. M. Kirschner and J. K. G. Watson, J. Mol. Spectrosc. 47, 347-350 (1973).

11. F. Michelot and J. Moret-Bailly, J. Phys. (Paris) 36, 451-460 (1975).

12. B. Zhilinskit, C.R. Acad. Sci, Paris, Ser. B 286, 135-137 (1978).

13. J. S. GRIfFith, "The Irreducible Tensor Method for Molecular Symmetry Groups,' Englewood Cliffs, N. J., 1962.

14. N. G. Rambidi, J. Mol. Struct. 28, 77-88 (1975).

15. B. I. Zhilinskil, V. A. Istomin, and N. F. Stepanov, in "Modern Aspects of Physical Chemistry," Vol. 10, Moscow Univ. Press, Moscow, 1978 (in Russian).

16. B. I. Zhilinskil, J. Mol. Struct. 46, 183-188 (1978).

17. J. Schwinger, in "Quantum Theory of Angular Momentum" (L. C. Biedenharn and H. Van Dam, Eds.), pp. 229-279, Academic Press, New York, 1965.

18. M. E. Rose, "Elementary Theory of Angular Momentum," Wiley, New York, 1957.

19. D. A. Varshalovich, A. N. MoscaleV, and V. K. Khersonskit, "Quantum Theory of Angular Momentum," Nauka, Leningrad, 1975 (in Russian).

20. B. Zhilinskil, G. Grenet, AND M. Kibler, in "Abstracts of VII International Colloquium on Group Theoretical Methods in Physics, Austin, Texas, Sept. 1978" ; B. ZhILINsKII, G. GreneT, AND M. KibLeR, to be published.

21. J. C. Hilico and M. Dang Nhu, J. Phys. (Paris) 35, 527-532 (1974).

22. J. C. Hilico, J. P. Champion, and G. Pierre, J. Mol. Spectrosc. 60, 422-425 (1976).

23. L. D. Landau and E. M. Lifshitz, "Quantum Mechanics," Pergamon, Oxford, 1958.

24. F. Michelot, J. Mol. Spectrosc. 63, 227-240 (1976).

25. J. C. Hilico, Thèse, Dijon, A.O. 3666 (1969).

26. B. Bobin and J. C. Hilico, J. Phys. (Paris) 36, 225-233 (1975); E. Pascaud, Thèse, Dijon, A.O. 6853 (1972); J. C. Hilico, J. Phys. (Paris) 31, 289 (1970).

27. A. Bauder, R. Meyer, and H. H. Günthard, Mol. Phys. 28, 1305-1343 (1974).

28. B. I. Zhilinskir and N. F. Stepanov, Zh. Koord. Khim. 4, 1292-1299 (1978) (in Russian). 
29. A. I. Boldirev, O. P. Charkin, N. G. Rambidi, and V. I. Avdeev, Chem. Phys. Lett. 44, 20-24 (1976).

30. V. P. SPIRIDonov, in "Modern Aspects of Physical Chemistry," Vol. 9, pp. 226-283, Moscow Univ. Press, Moscow, 1976 (in Russian).

31. B. I. Zhilinskit, V. A. Istomin, and N. F. Stepanov, in "Abstracts of the Third All-Union Symposium on Molecular Spectroscopy of High and Super-high Resolution, Novosibirsk, September, 1976."

32. V. A. Istomin, N. F. Stepanov, and B. I. ZhilinskiI, J. Mol. Spectrosc. 67, 265-282 (1977);

B. I. Zhilinskit, V. A. Istomin, and N. F. Stepanov, Chem. Phys. 31, 413-423 (1978).

33. A. R. Hoy and P. R. BUnker, J. Mol. Spectrosc. 52, 439-456 (1974); P. R. BUnKer ANd B. M. Landsberg, J. Mol. Spectrosc. 67, 374-385 (1977); S. L. Holmgren, M. Waldam, and W. KLEMPERer, J. Chem. Phys. 67, 4414-4422 (1977). 